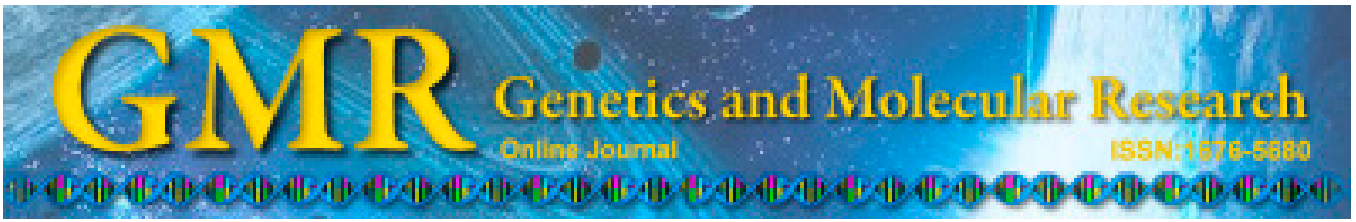

\title{
Relevance of E-cadherin expression to EGFR-TKI molecular targeted therapy sensitivity/resistance and its clinical significance
}

\author{
R.C. Xing ${ }^{1,2,3}$, J. Zheng ${ }^{1,2,3}$, W.H. Zheng ${ }^{4}$, Z.P. Qin ${ }^{5,6}$, W. Liu ${ }^{1}$ and R.C. Yao ${ }^{1,2,3}$ \\ ${ }^{1}$ Institute of Hepatopancreatobiliary Surgery, \\ China Three Gorges University, Yichang, Hubei, China \\ ${ }^{2}$ Department of General Surgery, The First College of Clinical Medical Science, \\ China Three Gorges University, Yichang, Hubei, China \\ ${ }^{3}$ Department of General Surgery, \\ Yichang Central People's Hospital, Yichang, Hubei, China \\ ${ }^{4}$ Department of Pharmacology, Medical Science College, \\ China Three Gorges University, Yichang Hubei, China \\ ${ }^{5}$ Department of Cardiothoracic Surgery, \\ People's Hospital of China Three Gorges University, Yichang, Hubei, China \\ ${ }^{6}$ Department of Cardiothoracic Surgery, \\ The First People's Hospital of Yichang, Hubei, China \\ Corresponding author: J. Zheng \\ E-mail: zhengjun1995@163.com
}

Genet. Mol. Res. 14 (2): 5785-5792 (2015)

Received August 26, 2014

Accepted January 21, 2015

Published May 29, 2015

DOI http://dx.doi.org/10.4238/2015.May.29.10

\begin{abstract}
We examined the effect of E-cadherin expression on epidermal growth factor receptor-tyrosine kinase inhibitor (EGFR-TKI) molecular targeted therapy sensitivity/resistance. We treated MCF-7, MDA-MB-231, T24, SiHa, H460, SK-HEP-1, MHCC97-H, and THP1 cells with the EGFR-TKIs PD153035 and gefitinib, and then tested the drug-resistance and sensitivity using the MTT method, calculated $\mathrm{IC}_{50}$ values for each cell line, and compared the results to E-cadherin content. The MTT assay was used to determine the survival rates of
\end{abstract}


MCF-7, MDA-MB-231, T24, SiHa, H460, SK-HEP-1, MHCC97-H, and THP-1 cells upon the action of EGFR-TKI (PD153035, gefitinib). For PD153035, the $\mathrm{IC}_{50}$ in MCF-7, MDA-MB-231, T24, and SiHa cells differed from that of H460, SK-HEP-1, MHCC97-H, and THP-1 (P < 0.05). Following gefitinib treatment, the $\mathrm{IC}_{50}$ values of MCF-7, MDAMB-231, T24, and SiHa cells differed from those of H460, SK-HEP-1, MHCC97-H, and THP-1 cells $(\mathrm{P}<0.01)$. The survival rate of MCF-7, MDA-MB-231, T24, and SiHa cells clearly decreased with increasing drug concentration, indicating the cells were sensitive to the drugs and that E-cadherin expression was positive; however, H460, SK-HEP-1, MHCC97-H, and THP-1 cells showed no significant decreased with increasing drug concentration, indicating that they were resistant to the drugs and that E-cadherin expression was negative. The survival rate of epithelial tumor cells through the action of EGFR-TKI is related to E-cadherin expression. E-cadherin may play a significant role in the sensitivity regulation of EGFR molecular targeting treatment. E-cadherin may provide important clues for selecting proper EGFRTKI molecular targeting treatment.

Key words: Clinical significance; E-cadherin; Molecular treatment; Epidermal growth factor receptor-tyrosine kinase inhibitor; Resistance

\section{INTRODUCTION}

With advances in the understanding of malignancy, molecular pharmaceutics have demonstrated promising methods for targeting cancer cell abnormal routes. The molecular targeting treatment for epidermal growth factor receptor (EGFR) has been widely applied for treating various tumors, which has unique advantages compared to conventional chemotherapy drugs. During tumor occurrence and development, during which EGFR overexpression or mutation is observed, over-activated transmission of signals simulated by epidermal growth factor (EGF) to cells results in unlimited cell proliferation and malignant transformation, causing local infiltration and distant metastasis. A number of studies have found that EGFR overexpression in cancer patients is closely related to the level of malignancy, infiltration, and metastasis and poor prognosis, and is an important molecular target (Saif, 2010; Fratto et al., 2011). E-cadherin not only plays a role in tumor tissue infiltration and metastasis, but also is closely related to EGFR molecular targeting treatment. The clinical significance and relevance of E-cadherin for EGFR molecular targeting treatment resistance/sensitivity were analyzed.

\section{MATERIAL AND METHODS}

\section{Sources of cells}

The MCF-7, MDA-MB-231, T24, SiHa, H460, SK-HEP-1, MHCC97-H, and THP-1 cells used in the experiment were donated by the Institute of Molecular Biology in Medical College of CTGU. 


\section{Experimental apparatus and reagents}

\section{Experimental apparatus}

$\mathrm{A} \mathrm{CO}_{2}$ constant temperature incubator was used (model: MCO175175C; SANYO, Osaka, Japan). The XS2-D2 inverted biological microscope was from Chongqing MIC Technology Co, Ltd. (Chongqing, China). The electric-heated thermostatic water bath, model: DKS26, was from Shanghai Jing Hong Laboratory Instrument Co., Ltd. (Shanghai, China). The SW-CQ-IF clean bench was from Shanghai Glass Instrument Plant No.1 (Shanghai, China). The LDZ5-2 auto-balancing centrifuge was from Beijing Medical Centrifuge Factory (Beijing, China). The ultra-low temperature freezer, model: ELT-13V, was from LANIS (Santa Monica, CA, USA). The negative glass filter, model: $1630950 \mathrm{~mm} / 47 \mathrm{~mm}$, was from Beijing Sartorius Company (Beijing, China).

\section{Reagents}

RPMI-1640 medium was from Gibco (Grand Island, NY, USA); penicillin and streptomycin were from North China Pharmaceutical Co., Ltd. (Hebei, China). Calf serum was from Hangzhou Sijiqing Bio-technology Co., Ltd. (Hangzhou, China). E-cadherin primary antibody mouse anti-human monoclonal antibody was from Cell Signaling Technology (Danvers, MA, USA). MTT reagent, the E-cadherin secondary goat-anti-mouse RAP antibody, $\beta$-actin primary rabbit anti-human antibody, and $\beta$-actin secondary goat-anti-rabbit RAP antibody were from Wuhan Guge Biotechnology Co., Ltd. (Wuhan, China). The BCA protein quantification kit was from Beyotime Biotechnology Co., Ltd. (Shanghai, China). PD153035 and gefitinib were from Nanjing Debao Biochemical Instrument Co., Ltd. (Jiangsu, China).

\section{Experimental methods}

\section{MTT method}

We tested the cellular proliferation inhibition rate on a 96-well tissue culture plate. The single-cell suspension was prepared and the cell concentration was adjusted to $2 \times 10^{5} /$ $\mathrm{mL}$. Next, $100 \mu \mathrm{L}$ cell suspension was added to the tissue culture plate, with $2 \times 10^{4}$ cells in each well, using 3 wells per group. After overnight incubation, the plate was turned over to empty the nutrient solution; nutrient solutions containing different drug concentrations were added to the cells and the plate was placed in a $\mathrm{CO}_{2}$ incubator for $48 \mathrm{~h}$. Next, $20 \mu \mathrm{L}$ MTT was added to each well and the plate was cultured for $2 \mathrm{~h}$ at $37^{\circ} \mathrm{C}$. The supernatant was discarded and $100 \mu \mathrm{L}$ dimethyl sulfoxide was added. Optical density (OD) was measured at a wavelength of $570 \mathrm{~nm}$ using a spectrophotometer. To calculate the cell proliferation survival rate, the following equation was used: Cell proliferation rate $(\%)=[(\mathrm{OD}$ of the experimental group-background OD) / OD of the control group] x 100\%.

\section{Western blotting}

Protein samples were extracted and quantified, and $50 \mu \mathrm{g}$ each protein was added to a polyacrylamide gel for conventional electrophoresis and transfer to a nitrocellulose mem- 
brane. The nitrocellulose film was sealed with phosphate-buffered saline containing $5 \%$ skim milk and incubated at room temperature for 60 min. Primary antibody diluted 1:1000 was added and the membrane was placed on a shaking table at $4^{\circ} \mathrm{C}$ overnight. The following day, the membrane was washed with phosphate-buffered saline 4 times. Secondary antibody diluted 1:5000 was added and the membrane was placed on a shaking table for $60 \mathrm{~min}$. The membrane was washed 4 times with phosphate-buffered saline. ECL substrate was added, the membrane was incubated for $5 \mathrm{~min}$, and the membrane was developed in a dark room with $\beta$-actin used as an internal reference.

\section{Statistical analysis}

All data were processed using the Excel and the SPSS13.0 software package (SPSS, Inc., Chicago, IL, USA) and the data were statistically analyzed.

\section{RESULTS}

\section{MTT results}

MTT was used to measure the survival rate of MCF-7, MDA-MB-231, T24, SiHa, H460, SK-HEP-1, MHCC97-H, and THP-1 upon the action of EGFR-TKI (PD153035). According to the results, the survival rate of MCF-7, MDA-MB-231, T24, and SiHa decreases with the increase of concentration, which means they are sensitive to the drug; while that of H460, SK-HEP-1, MHCC97-H, and THP-1 showed no clear decrease with increasing concentration, indicating that the cells were drug-resistant (Figure 1).

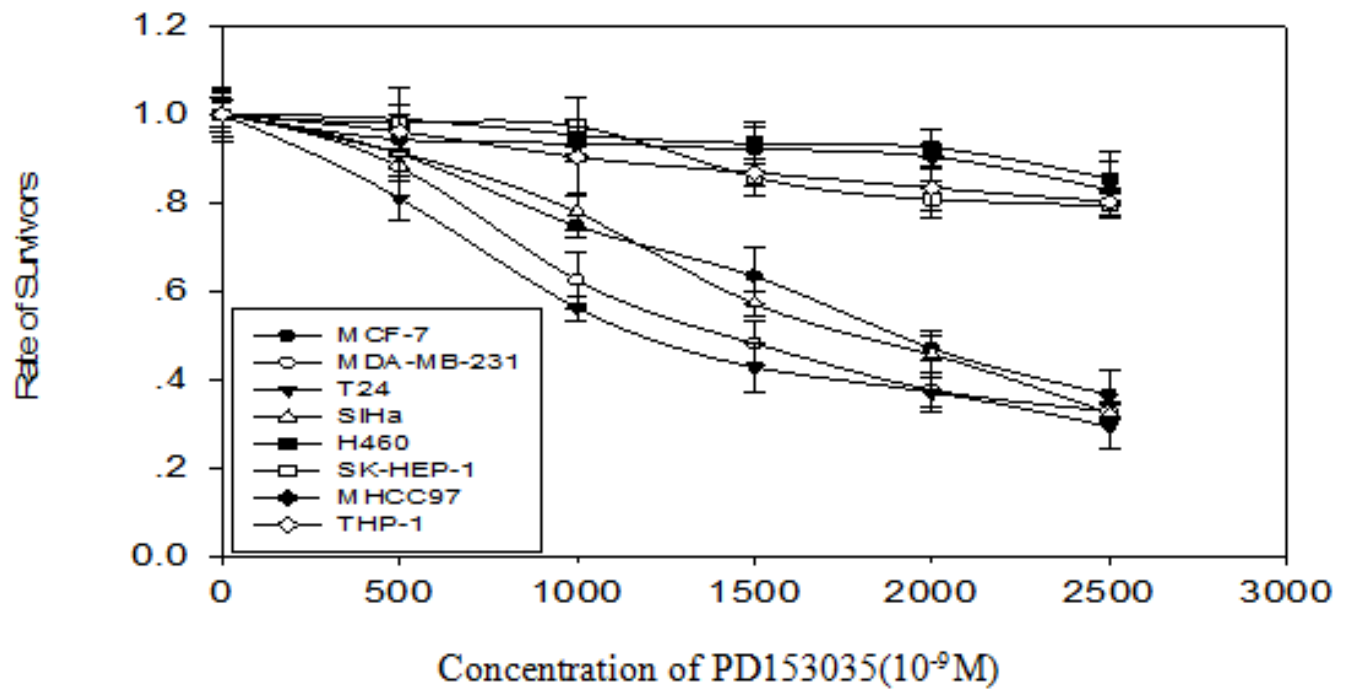

Figure 1. Survival curve of 8 cell lines following treatment with EGFR-TKI (PD153035) measured by MTT assay. 
The MTT assay was used to measure the survival rate of MCF-7, MDA-MB-231, T24, SiHa, H460, SK-HEP-1, MHCC97-H, and THP-1 cells in the presence of EGFR-TKI (gefitinib). Based on our results, the survival rate of MCF-7, MDA-MB-231, T24, and SiHa cells decreased with increasing EGFR-TKI concentration, indicating that these cells were sensitive to the drug. The H460, SK-HEP-1, MHCC97-H, and THP-1 cells showed no clear decline with increasing EGFR-TKI concentration, indicating that these cells were resistant to the drug (Figure 2).

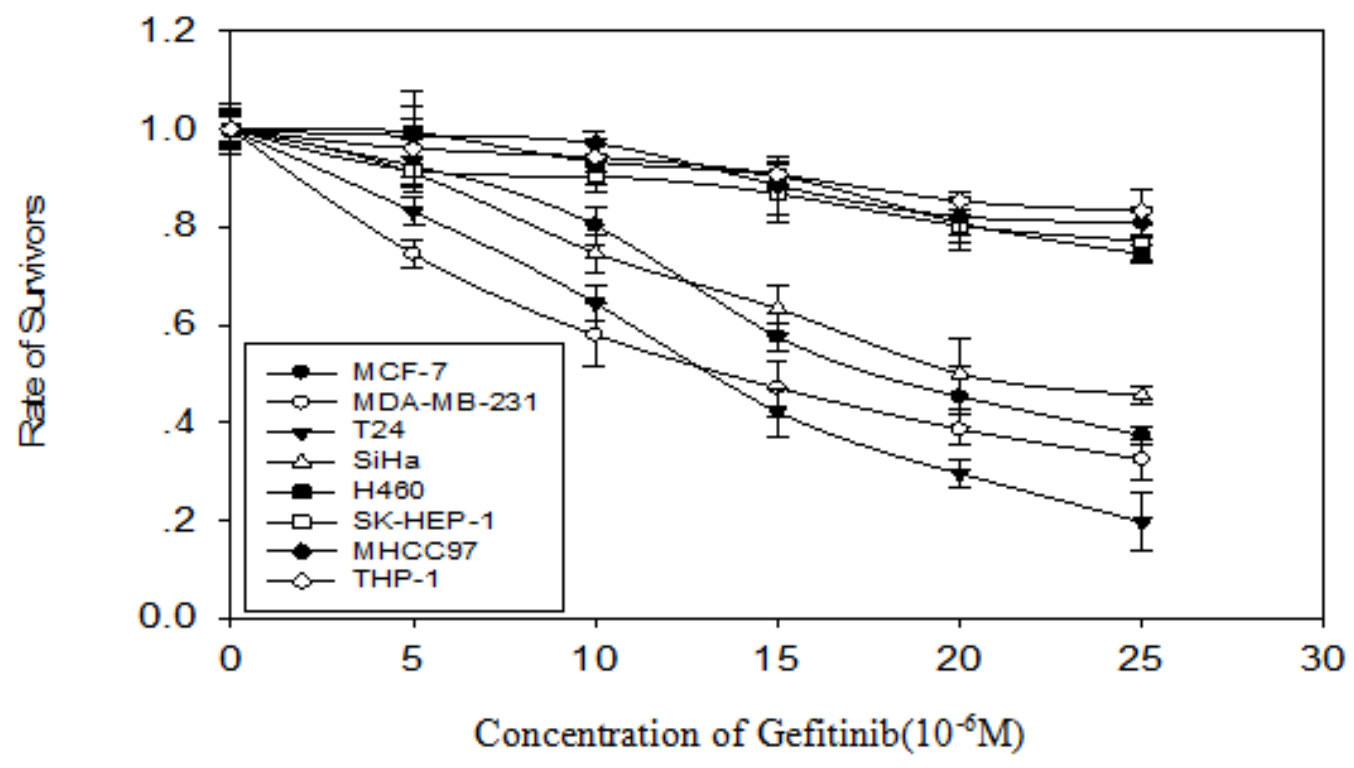

Figure 2. Survival curve of 8 cell lines following treatment with EGFR-TKI (gefitinib) measured by MTT assay.

The concentration of an inhibitor where the response was reduced by half $\left(\mathrm{IC}_{50}\right)$ for the 8 cell lines treated with EGFR-TKI (PD153035, gefitinib) was calculated and that of epithelial cells was compared to that of mesenchymal cells. According to the results, in the presence of PD153035, the $\mathrm{IC}_{50}$ of MCF-7, MDA-MB-231, T24, and SiHa differed from those of H460, SK-HEP-1, MHCC97-H, and THP-1 ( $<0.05)$; following treatment with gefitinib, the $\mathrm{IC}_{50}$ in MCF-7, MDA-MB-231, T24, and SiHa cells differed from that in H460, SK-HEP-1, MHCC97-H, and THP-1 $(\mathrm{P}<0.01)$, as is shown in Table 1.

Table 1. Treatment with EGFR-TKI(PD153035, gefitinib) corresponding $\mathrm{IC}_{50}$ value concentration of 8 cell lines.

\begin{tabular}{llcc}
\hline Kind & Name & \multicolumn{2}{c}{ IC50 $(\mathrm{mM})$} \\
\cline { 3 - 4 } & & PD153035 & Gefitinib \\
\hline Epithelial cells & MCF-7 & 1.94 & 1.87 \\
& MDA-MB-231 & 1.52 & 1.45 \\
& T24 & 1.40 & 1.38 \\
Mesenchymal cells & SiHa & 1.85 & 2.01 \\
& H460 & 12.22 & 5.53 \\
& SK-HEP-1 & 5.18 & 5.50 \\
$t$ test & MHCC97 & 11.32 & 5.72 \\
P value & THP-1 & 5.84 & 5.72 \\
& & 3.81 & 9.62 \\
& & 0.031 & 0.001 \\
\hline
\end{tabular}




\section{Western blotting}

Western blotting analysis results showed that E-cadherin in MCF-7, MDA-MB-231, T24, and SiHa showed positive expression, while E-cadherin in H460, SK-HEP-1, MHCC97$\mathrm{H}$, and THP-1 showed negative expression, as shown in Figure 3.

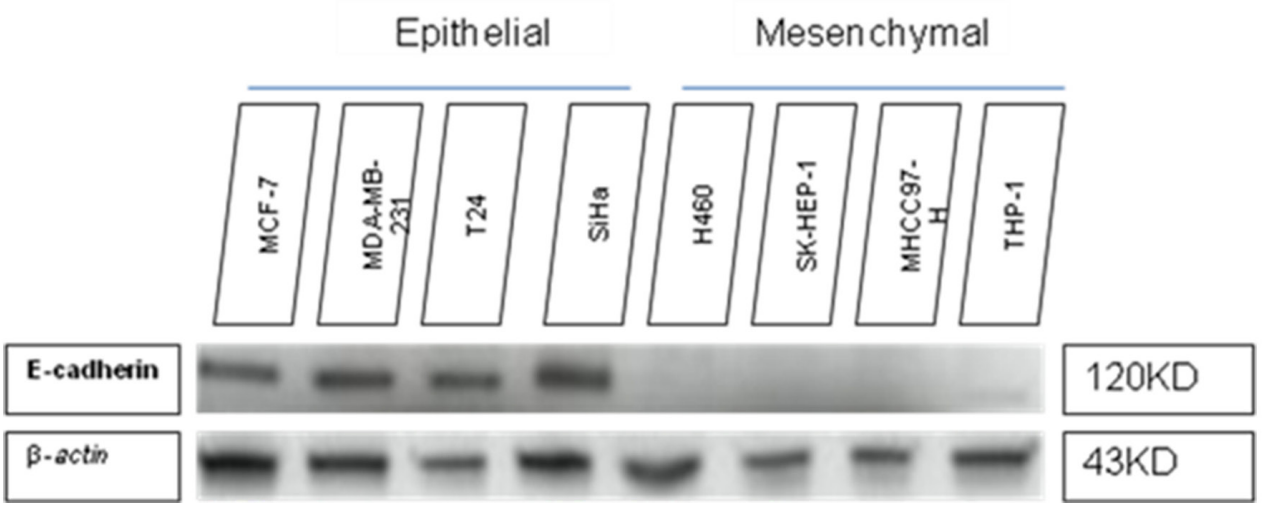

Figure 3. Western blot results of 8 cell lines.

\section{DISCUSSION}

E-cadherin is a kind of calcium-dependent transmembrane protein with intercellular adhesion capabilities, and is located in various epithelial cells in humans and animals. This protein plays an important role in maintaining cell polarity and integrity. The intracellular region of E-cadherin is highly conserved, forming complexes with catenin to connect to the actin cytoskeleton, forming and maintaining the secure junction between cells and normal tissue structure (Tunggal et al., 2005). E-cadherin is an important protein that enhances the adhesion between epithelial cells and maintains tissue structure stability; its expression is subject to genetic control, signal pathways, zinc finger protein family, and miRNAs, etc. (Baranwal and Alahari, 2009). This protein plays an important role in signal transduction to mediate cells and recruit immune response cells. Studies have shown that E-cadherin expression is closely related to molecular targeting treatment resistance/sensitivity of tumor EGFR. E-Cadherin may participate in the development of EGFR-TKI sensitivity or resistance, while playing a role in the intercellular complex regulation. In this study, MTT was used to measure the survival rate of MCF-7, MDA-MB-231, T24, SiHa, H460, SK-HEP-1, MHCC97-H, and THP-1 cells in the presence of EGFR-TKI (PD153035, gefitinib). According to our results, the survival rate of MCF-7, MDA-MB-231, T24, and SiHa cells decreased with increasing concentration, indicating that these cells were sensitive to the drugs, while that of H460, SK-HEP-1, MHCC97-H, and THP-1 cells showed no clear decrease with increasing concentration, indicating that these cells were resistant to the drug (Figures 1 and 2).

The epithelial mesenchymal transition is one of the important mechanisms of tumor tissue infiltration and metastasis, which is mainly characterized by the loss of epithelial type E-cadherin expression. This is consistent with the positive expression of E-cadherin in epithelial carcinoma cells (MCF-7, MDA-MB-231, T24, and SiHa) and negative expression in mesenchymal carcinoma cells (H460, SK-HEP-1, MHCC97-H, and THP-1). The decrease or 
loss of E-cadherin expression is related not only to the occurrence and development of tumors but also to poorly differentiated, highly aggressive, and metastatic tumors, and can directly influence patient prognosis. One of the major functions of E-cadherin is to mediate the adhesion between homotype cells, and this protein plays a role in the embryonic development, formation of normal tissue epithelial layer, and maintenance of their integrity and polarity, which may prohibit tumor metastasis and invasion.

In this study, for the survival rate of MCF-7, MDA-MB-231, T24, SiHa, H460, SKHEP-1, MHCC97-H, and THP-1 cells treated with EGFR-TKI (PD153035, gefitinib), the $\mathrm{IC}_{50}$ values of MCF-7, MDA-MB-231, T24, and SiHa cells differed from those of H460, SKHEP-1, MHCC97-H, and THP-1 $(\mathrm{P}<0.05)$. Following treatment with gefitinib, the $\mathrm{IC}_{50}$ values of MCF-7, MDA-MB-231, T24, and SiHa cells differed from those of H460, SK-HEP-1, MHCC97-H, and THP-1 cells $(\mathrm{P}<0.01)$. As is shown in Figure 1-3, the survival rate of MCF7, MDA-MB-231, T24, and SiHa cells clearly decreased as the concentration of the 2 drugs increased, indicating they were sensitive to the drugs and that E-cadherin expression was positive. However, that of H460, SK-HEP-1, MHCC97-H, and THP-1 showed no significant decrease with increasing drug concentration, indicating that they were resistant to the drugs and that E-cadherin expression was negative. This suggests that a clear relationship exists between E-cadherin expression and tumor resistance/sensitivity to EGFR-TKI, and E-cadherin expression may play an important role in this process. As a marker, E-cadherin can provide important clues in selecting proper cases for EGFR-TKI molecular targeting treatment.

There may be a close relationship between E-cadherin expression and EGFR-TKI molecular targeting treatment sensitivity/resistance. Subcellular localization of EGFR and Ecadherin is very similar, and both exist in the intercellular zonula adherens. Previous studies found that a 2-way interaction exists between EGFR and E-cadherin (Andl and Rustgi, 2005).

When EGFR regulates E-cadherin, the intracytoplasm of E-cadherin connects to the core of $\beta$-catenin to recruit compounds and then connect to the actin cytoskeleton, maintaining the tight junction between cells. $\beta$-Catenin is the substrate of EGFR. When any EGFR ligand (such as EGF, transforming growth factor- $\beta$ ) combines with EGFR, intracytoplasm EGFR with thymidine kinase activity is activated, resulting in phosphorylation between E-cadherin and $\beta$-catenin in $\beta$-catenin compounds so that $\beta$-catenin is dissociated from compounds and the compounds from actin, reducing intercellular adhesion. Therefore, binding of the ligand to the EGFR receptor may cause downregulation of E-cadherin expression and initiate the epithelial mesenchymal transition (Yasmeen et al., 2006).

E-cadherin is a calcium-dependent transmembrane protein capable of intercellular adhesion, and EGFR of E-cadherin may be activated for phosphorylation when the cellular calcium concentration increases (Pece and Gutkind, 2000; Heijinki et al., 2007). In addition, E-cadherin can bind to EGFR receptors through its extracellular domain to reduce the activity of these receptors as well as EGF binding affinity (Qian et al., 2004).

Recent studies have revealed that EGFR phosphorylation and internalization were significantly enhanced with E-cadherin mutations (Bremm et al., 2008). However, there have been no studies to examine the influence of the normal functions, lack, or mutation of E-cadherin on EGFR-targeted treatment sensitivity. Stimulated by growth factors such as EGF, the rate of EGFR internalization increases, while mechanochemical degradation proteins are recruited to accelerate degradation. These may be the mechanism by which EGF signal strength and continuity is controlled in the body. Additional studies are needed to determine the specific signal path of mutual regulation between E-cadherin and EGFR. 


\section{REFERENCES}

Andl CD and Rustgi AK (2005). No one-way street: cross-talk between E-cadherin and receptor tyrosine kinase (RTK) signaling: a mechanism to regulate RTK activity. Cancer Biol. Ther. 4: 28-31.

Baranwal S and Alahari SK (2009). Molecular mechanisms controlling E-cadherin expression in breast cancer. Biochem. Biophys. Res. Commun. 384: 6-11.

Bremm A, Walch A, Fuchs M, Mages J, et al. (2008). Enhanced activation of epidermal growth factor receptor caused by tumor-derived E-cadherin mutations. Cancer Res. 68: 707-714.

Fratto ME, Santini D, Vincernzi B, Silvestris N, et al. (2011). Targeting EGFR in bilio-pancreatic and liver carcinoma. Front. Biosci. (Schol. Ed.) 3: 16-22.

Heijinki H, Kies PM, Kauffman HF, Postma DS, et al. (2007). Down-regulation of E-cadherin in human bronchial epithelial cells leads to epidermal growth factor receptor-dependent Th2 cell-promoting activity. J. Immunol. 178: 7678-7685.

Pece S and Gutkind JS (2000). Signaling from E-cadherins to the MAPK pathway by the recruitment and activation of epidermal growth factor receptors upon cell-cell contact formation. J. Biol. Chem. 275: 41227-41233.

Qian X, Karpova T, Sheppard AM, McNally J, et al. (2004). E-cadherin-mediated adhesion inhibits ligand-dependent activation of diverse receptor tyrosine kinases. EMBO J. 23: 1739-1748.

Saif MW (2010). Colorectal cancer in review: the role of the EGFR pathway. Expert Opin. Investig. Drugs 19: 357-369.

Tunggal JA, Helfrich I, Schmitz A, Schwarz H, et al. (2005). E-cadherin is essential for in vivo epidermal barrier function by regulating tight junctions. EMBO J. 24: 1146-1156.

Yasmeen A, Bismap TA and Al Moustafa AE (2006). ErbB receptors and epithelial-cadherin-catenin complex in human carcinomas. Future Oncol. 2: 765-781. 